\title{
McConnell sign in a patient with massive acute pulmonary embolism
}

\author{
Waiel Abusnina MD, Hazim Bukamur MD, Fuad Zeid MD
}

\section{CASE}

A 69-year-old man with a history of hypertension and diabetes was admitted to the hospital for lower extremity weakness and recurrent falls of one month's duration. Magnetic resonance imaging of the brain showed a $6 \times 3 \times 4 \mathrm{~cm}$ brain mass. Fifteen days prior to his admission, the patient underwent stereotactic brain biopsy with a right frontal craniotomy. He arrived from the nursing home with an altered mental status, was intubated in the emergency room, and was admitted to the intensive care unit (ICU). He had a heart rate of 140 beats per minute and a blood pressure of 90/60 mm Hg. Laboratory workup revealed WBC 11.9 $10^{9} / \mathrm{L}$, lactic acid $4.1 \mathrm{mmol} / \mathrm{L}$, and troponin $1.2 \mathrm{ng} / \mathrm{mL}$. His electrocardiogram showed a sinus tachycardia but otherwise was within normal limits. Differential diagnosis at this time included septic shock and NSTEMI with cardiogenic shock. A central line was inserted, intravenous normal saline boluses were given, and the patient was started on broad spectrum antibiotics. Transthoracic echocardiogram was performed and revealed marked right ventricular (RV) dilation and a relatively small left ventricular chamber. McConnell's sign was noted (Video 1, Figure 1). The RV systolic pressure was $41 \mathrm{mmHg}$, the RVGLS was $5 \%$, and the $\mathrm{RV}$ annulus $\mathrm{S}$ wave was $5.37 \mathrm{~cm} / \mathrm{sec}$. Doppler ultrasound of the lower extremities showed a non-occlusive thrombus in the left popliteal vein. Computed tomography of the chest showed extensive bilateral pulmonary emboli with a saddle embolus extending across the main pulmonary arterial bifurcation (Figure 2). The patient was started on a heparin drip; however, given his recent cranial surgery, the decision was made not to start systemic or catheter directed thrombolysis. After a prolonged hospital stay, the patient was eventually discharged to a nursing home.

Corresponding author: Hazim Bukamur

Contact Information: Hazimbukamur@gmail.com DOI: 10.12746/swrccc.v8i35.725

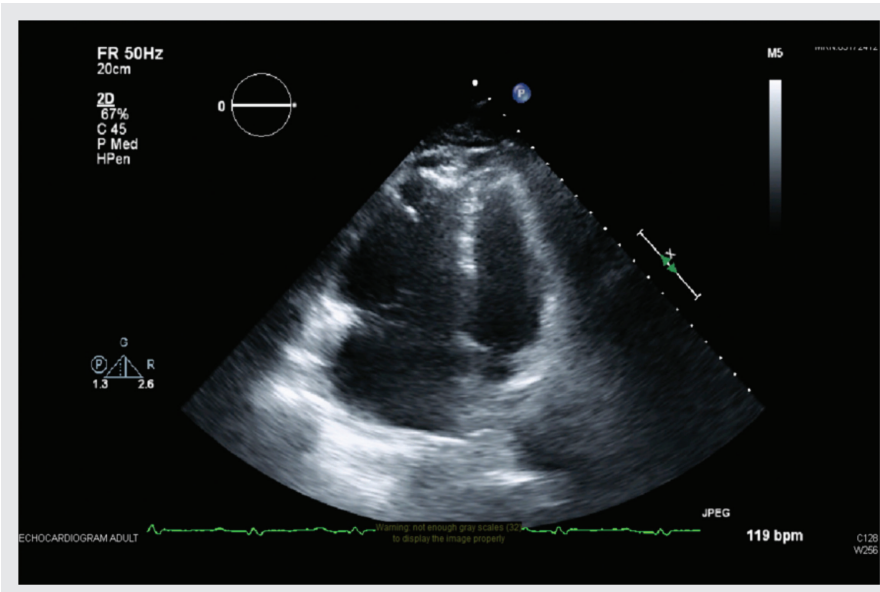

Figure 1. Echocardiographic image showing dilatation of the right ventricle and no movement of the right lateral ventricle wall in comparison to the left ventricle.

\section{Discussion}

Pulmonary embolism (PE) can result in a catastrophic event with significant morbidity and mortality, especially when associated with RV dysfunction. ${ }^{1,2}$ However, it is still often unsuspected and underdiagnosed. Depending on clinical presentation, the case

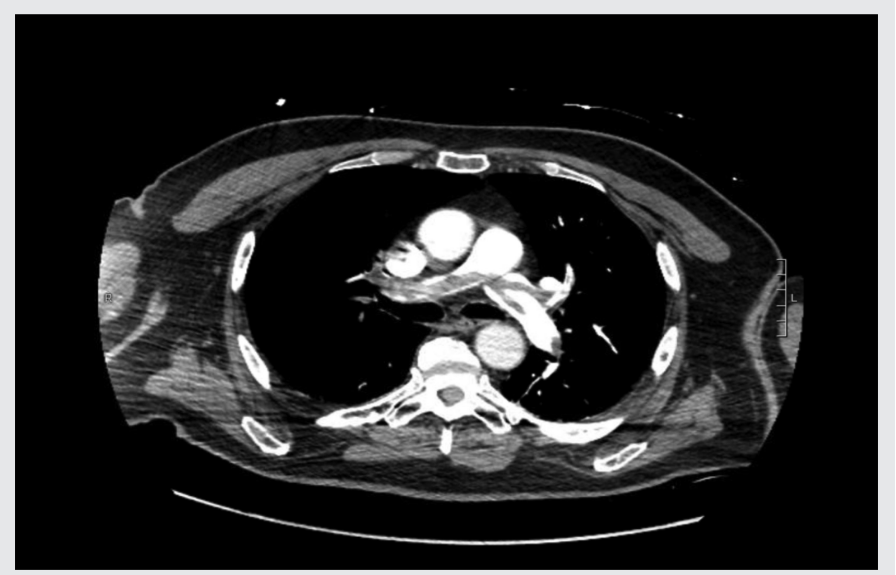

Figure 2. Computed tomography with contrast reveals a large saddle embolus. 
fatality rate for acute PE ranges from less than $1 \%$ up to $60 \% .{ }^{3}$ Pulmonary embolism should be suspected in all patients who present with new or worsening dyspnea, chest pain, or sustained hypotension without an alternative or obvious cause. The diagnosis, however, is confirmed by objective testing in only about $20 \%$ of patients. ${ }^{4}$ In acutely unstable patients, echocardiography can be highly suggestive of PE. Submassive PE has been suggested when RV hypokinesia is identified in otherwise hemodynamically stable patients. ${ }^{5}$

Echocardiographic RV dysfunction is indicative of a poor prognosis, and patients with this finding are at risk for subsequent clinical worsening and PE-related death. These patients may benefit from more aggressive therapeutic strategies, including thrombolytic therapy. 6,7 "McConnell's sign," defined as RV free wall hypokinesis in the presence of normal RV apical contractility, has $77 \%$ sensitivity and $94 \%$ specificity for the diagnosis of acute PE with a positive predictive value of $71 \%$ and a negative predictive value of $96 \%{ }^{8}$ Casazza et al. demonstrated that McConnell's sign can also be seen in cases of RV infarction and thus cannot be considered pathognomonic for acute PE. ${ }^{9} \mathrm{~A}$ pulmonary perfusion defect of at least $25 \%$ (moderate degree) is required for McConnell's sign to be demonstrated by transthoracic echocardiogram. ${ }^{10}$

Our patient had extensive bilateral pulmonary emboli and a saddle embolus leading to severe RV mid-free wall hypokinesis. Other parameters, including apical contractility, were otherwise within normal limits. Coupling the imaging findings with sudden cardiopulmonary decompensation increased our suspicion for submassive PE, which was confirmed by CT angiography of the chest. Normalization of the regional RV free wall dysfunction with thrombolytic therapy has been demonstrated in patients with massive PE. ${ }^{11}$ Our patient was not a candidate for thrombolytic therapy as he was at high risk for major hemorrhage due to a brain neoplasm and recent brain surgery. ${ }^{12}$

Recognition of McConnell's sign and understanding that its pathogenesis involves a moderate-sized pulmonary perfusion defect provide critical care physicians additional information to reach an earlier diagnosis and improve outcomes in cases of acute pulmonary embolism. sign.

Video 1: Echocardiogram demonstrating McConnell

Video 2: Echocardiogram showing significant dilatation of the right ventricle with reduced right ventricular systolic function, flattened septum, large mobile vegetation of uncertain etiology on the tricuspid valve, and McConnell sign.

Keywords: pulmonary emboli, right ventricular dysfunction, McConnell's sign

Article citation: Abusnina W, Bukamur H, Zeid F. McConnell sign in a patient with massive acute pulmonary embolism. The Southwest Respiratory and Critical Care Chronicles 2020;8(35):77-79

From: Department of Internal medicine, Marshall University, Joan C. Edwards School of Medicine, Huntington, WV

Submitted: 4/16/2020

Accepted: 6/22/2020

Reviewer: Pooja Sethi MD, Aliakbar Arvandi MD

Conflicts of interest: none

This work is licensed under a Creative Commons

Attribution-ShareAlike 4.0 International License.

\section{REFERENCES}

1. McIntyre KM, Sasahara AA. The hemodynamic response to pulmonary embolism in patients without prior cardiopulmonary disease. Am J Cardiol 1971;28(3):288-94.

2. Grifoni S, Olivotto I, Cecchini P, et al. Short-term clinical outcome of patients with acute pulmonary embolism, normal blood pressure, and echocardiographic right ventricular dysfunction. Circulation [Internet] 2000;101(24):2817-22.

3. Goldhaber SZ, Visani L, De Rosa M. Acute pulmonary embolism: clinical outcomes in the International Cooperative Pulmonary Embolism Registry (ICOPER) [see comment]. Lancet 1999;353(9162):1386-9.

4. Righini M, Le Gal G, Aujesky D, et al. Diagnosis of pulmonary embolism by multidetector $\mathrm{CT}$ alone or combined with venous ultrasonography of the leg: a randomised noninferiority trial. Lancet 2008;371:1343-52.

5. Torbicki A, Perrier A, Konstantinides S, et al. Guidelines on the diagnosis and management of acute pulmonary embolism. Eur Heart J 2008;29(18):2276-315. 
6. Come PC, Kim D, Parker JA, et al. Early reversal of right ventricular dysfunction in patients with acute pulmonary embolism after treatment with intravenous tissue plasminogen activator. J Am Coll Cardiol [Internet] 1987;10(5):971-8.

7. Kucher N, Rossi E, De Rosa M, et al. Prognostic role of echocardiography among patients with acute pulmonary embolism and a systolic arterial pressure of $90 \mathrm{~mm} \mathrm{Hg}$ or higher. Arch Intern Med 2012;165(15):1777-81.

8. McConnell MV, Solomon SD, Rayan ME, et al. Lee RT. Regional right ventricular dysfunction detected by echocardiography in acute pulmonary embolism. Am J Cardiol 1996; 78(4):469-73.

9. Casazza F, Bongarzoni A, Capozi A, et al. Regional right ventricular dysfunction in acute pulmonary embolism and right ventricular infarction. Eur J Echocardiogr 2005;6(1): $11-4$.

10. Kjaergaard J, Schaadt BK, Lund JO, et al. Quantification of right ventricular function in acute pulmonary embolism: Relation to extent of pulmonary perfusion defects. Eur J Echocardiogr 2008;9(5):641-5.

11. Kjaergaard J, Sogaard P, Hassager C. Right ventricular strain in pulmonary embolism by Doppler tissue echocardiography. J Am Soc Echocardiogr 2004;17(11):1210-2.

12. Kearon $\mathrm{C}, \mathrm{Akl}$ EA, Comerota AJ, et al. Antithrombotic therapy for VTE disease: Antithrombotic therapy and prevention of thrombosis, 9th ed: American College of Chest Physicians evidence-based clinical practice guidelines. Chest 2012;141 (2 SUPPL.):419-94. 